\title{
Research on the Single Neuron PID Control for Welding Bath Depth Xi-Wen LIU ${ }^{\mathrm{a}}$ and Chao-Ying LIU \\ Department of Electronic Information \& Mechanical Engineering, Zhaoqing University,Zhaoqing, Guangdong 526061, China \\ a Corresponding author: liuxiwen1111@163.com
}

Keywords: Welding bath depth, Intelligent control, Single-neuron PID, Hebb learning algorithm.

\begin{abstract}
Welding bath depth control system based on single neuron self-tuning PID is designed, and several learning algorithms for neuron weights are simulated, the simulation results show that this controller reacts quickly and has good stability. The experiment with various cross-section and seam-gap workpiece is perfect, showing that single-neuron self-tuning PID controller is suitable to the complicated welding system.
\end{abstract}

\section{Introduction}

With the rapid development of modern industry, higher and higher welding requirements should be met, and welding automation is essential to realize these requirements. The bath depth control is an important part of welding automation. As welding is a time-varying and nonlinear procedure with multi-factors acting on interactively, the controller's design way provided by classical control theory is not suitable to such complicated procedures as welding [1].

Since the birth of traditional PID method, it is widely used in practice. Some scholars had applied it to some kinds of welding successively previously [2]. But the traditional PID method has poor flexibility because its parameters are changeless, when it is applied to such complicated systems as welding, good results can't be obtained in most cases. As artificial neural network has strong ability of approaching and self-adapting, it is widely used in various control issues. In order to avoiding the shortcomings of traditional PID, artificial neural network and PID are combined for control, therefore, neural network PID controller comes into being. Single neuron PID is one of the simplest neural network PID controller. It has simple structure and costs little time for calculation, additionally, it has strong self-tuning ability. All these characteristics make it suitable to real-time control. Therefore, that how to apply single neuron self-tuning PID to welding bath depth control is discussed in this paper, and simulation with various learning algorithm is carried out, experiments show that good results can be gotten by using single neuron PID controller.

\section{Traditional PID and Single Neuron PID}

\section{Traditional PID Controller}

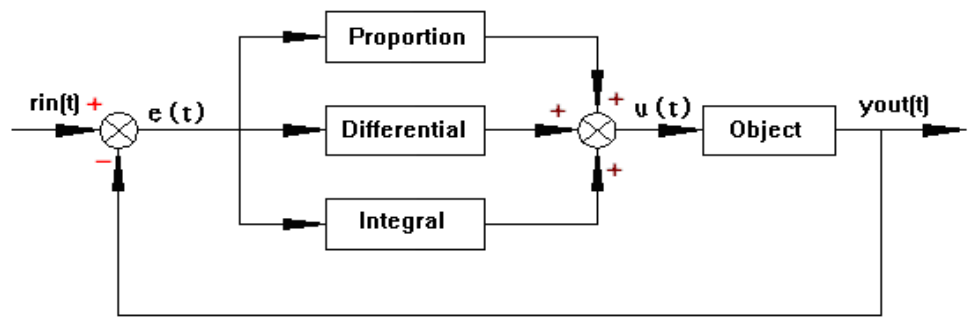

Fig.1 Traditional PID control system

Fig.1 shows the basic structure of traditional PID control system, as computer can only process discrete signals, the digit PID is expressed as 


$$
\begin{gathered}
u(k)=u(k-1)+K_{P}[e(k)-e(k-1)]+K_{I} e(k) \\
+K_{D}[e(k)-2 e(k-1)+e(k-2)]
\end{gathered}
$$

In the above equation, $K_{P}, K_{I}$ and $K_{D}$ are proportion coefficient, integral coefficient and differential coefficient respectively, $e$ represents error.

\section{Single-neuron PID Controller}

The basic structure of single neuron PID control system is as follows.

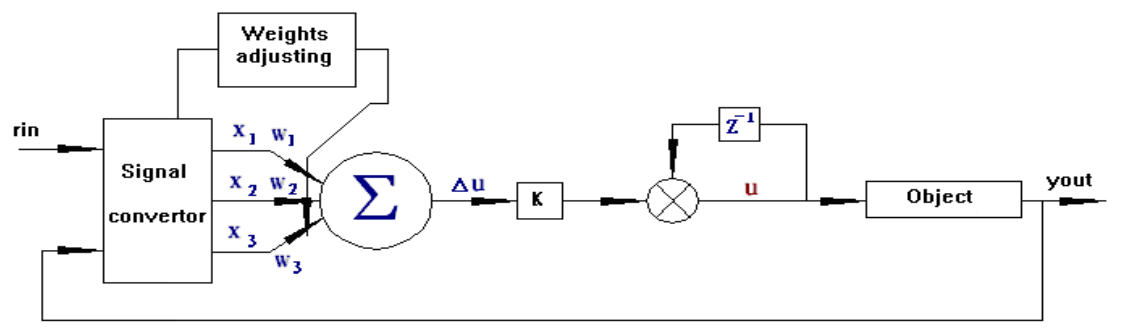

Fig.2 Single neuron PID control system

As shown in Fig.2, the neuron's input vector is $X=\left\{x_{1}(k), x_{2}(k), x_{3}(k)\right\}, x_{1}(k), x_{2}(k)$ and $x_{3}(k)$ are proportion error, integral error and differential error at the $k$-th time respectively. The corresponding weights of neuron's input vector are $w_{1}(k), w_{2}(k), w_{3}(k)$. In order to preventing the weights from increasing infinitely in learning, it is necessary to normalize it.

$$
w_{i}^{\prime}(k)=w_{i}(k) / \sum_{i=1}^{3}\left|w_{i}(k)\right|
$$

The neuron's output $u(k)$ is

$$
u(k)=u(k-1)+K \sum_{i=1}^{3} w_{i}^{\prime}(k) x_{i}(k)
$$

Suppose $e$ is error, therefore, in the above equation,

$$
\begin{aligned}
& x_{1}(k)=e(k)-e(k-1) \\
& x_{2}(k)=e(k) \\
& x_{3}(k)=e(k)-2 e(k-1)+e(k-2)
\end{aligned}
$$

$K$ is called neuron proportion coefficient, and it is very important. The bigger $K$ is, the better rapidity the control system has. But when $K$ is big, the overshoot is also big, and the controller may become unsteady. When the time delay of controlled object becomes bigger, $K$ must be decreased to ensure system stable. Therefore, it is important to decide a suitable $K$.

\section{Single neuron PID weights adjust algorithm}

According to the learning theory of neural network, there are several typical weights-learning algorithms as follows [6].

1) Non-supervised Hebb learning rule

$$
\left\{\begin{array}{l}
w_{1}(k)=w_{1}(k-1)+\eta_{P} x_{1}(k-1) u(k-1) \\
w_{2}(k)=w_{2}(k-1)+\eta_{I} x_{2}(k-1) u(k-1) \\
w_{3}(k)=w_{3}(k-1)+\eta_{D} x_{3}(k-1) u(k-1)
\end{array}\right.
$$

2) Supervised Delta learning rule 


$$
\left\{\begin{array}{l}
w_{1}(k)=w_{1}(k-1)+\eta_{P} e(k-1) u(k-1) \\
w_{2}(k)=w_{2}(k-1)+\eta_{I} e(k-1) u(k-1) \\
w_{3}(k)=w_{3}(k-1)+\eta_{D} e(k-1) u(k-1)
\end{array}\right.
$$

3) Supervised Hebb learning rule

$$
\left\{\begin{array}{l}
w_{1}(k)=w_{1}(k-1)+\eta_{P} e(k-1) x_{1}(k-1) u(k-1) \\
w_{2}(k)=w_{2}(k-1)+\eta_{I} e(k-1) x_{2}(k-1) u(k-1) \\
w_{3}(k)=w_{3}(k-1)+\eta_{D} e(k-1) x_{3}(k-1) u(k-1)
\end{array}\right.
$$

\section{4) Improved Hebb learning rule}

Experiences show that the learning parameters of PID mainly have relations with $e(k)$ and $\Delta e(k)$, therefore, the weights learning algorithm of single neuron PID can be improved as

$$
\left\{\begin{array}{l}
w_{1}(k)=w_{1}(k-1)+\eta_{P} e(k-1) u(k-1)[e(k)+\Delta e(k)] \\
w_{2}(k)=w_{2}(k-1)+\eta_{I} e(k-1) u(k-1)[e(k)+\Delta e(k)] \\
w_{3}(k)=w_{3}(k-1)+\eta_{D} e(k-1) u(k-1)[e(k)+\Delta e(k)]
\end{array}\right.
$$

In above learning algorithms, $\eta_{P}, \eta_{I}$ and $\eta_{D}$ are proportion learning speed, integral learning speed and differential learning speed. Generally, in control system, the most important factor is error, next is the error's first order difference, and the last is the error's second order difference. Therefore, every learning speed should be adjusted according to its significance.

\section{Welding Bath Depth Controller}

As welding current is the most important factor which affects bath depth, it is used to adjust bath depth. In non-penetration welding, it is difficult to acquire bath depth directly; generally, bath depth is estimated according to the welding conditions and bath surface information which is acquired through vision system. The vision system is often made up of CCD and light filters [6], and BP network is usually used as estimating tool.

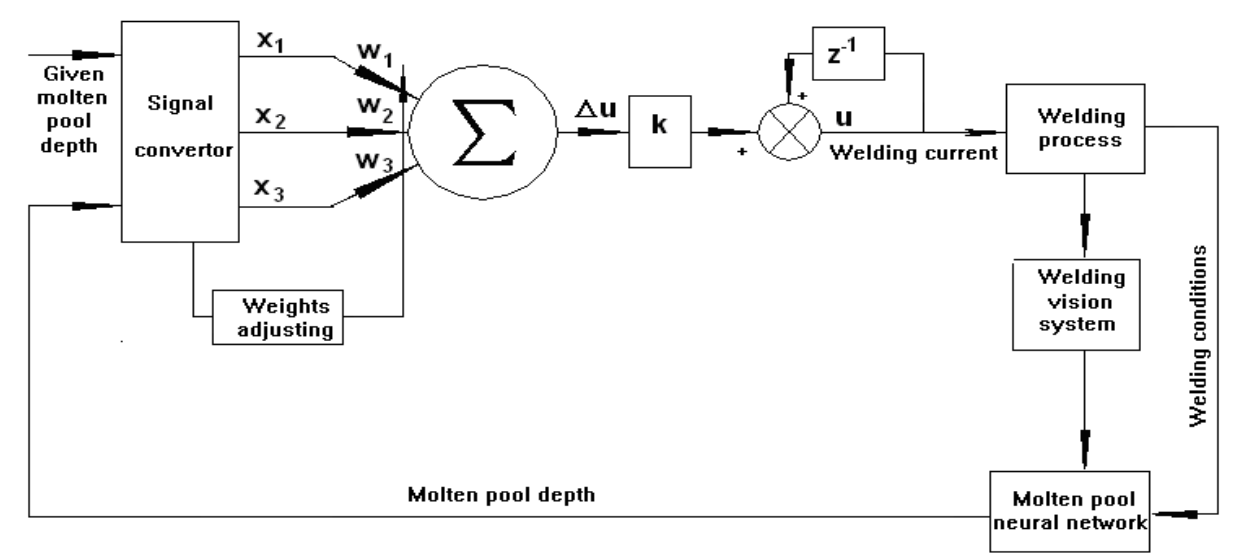

Fig.3 Single neuron PID bath depth control system

The structure of single-neuron bath depth PID control system is as shown in Fig.3. The input vector $X=\left\{x_{1}(k), x_{2}(k), x_{3}(k)\right\}$ can be gotten from signal convertor. The neuron outputs welding current, and the molten pool depth neural network calculates the depth according to welding conditions and information gotten from vision system.

In the modelling of welding bath depth neural network, many samples are used for training. It is essential to mention that unexpected errors will occur when the control current is beyond the scope 
of training samples. Therefore, when the current in control process is less than the least current of training samples, it should be set to equal to the least current of training samples. And when the current in control process is bigger than the maximal current of training samples, it should be set to equal to the maximal current of training samples.

\section{Controller Simulation}

The destination of simulation is to get proper parameters of controller, and to verify feasibility of control system. In this paper, simulations by using traditional PID and single-neuron PID with various learning algorithms are carried out to compare their performance. First, proper proportion coefficient, integral coefficient and differential coefficient of traditional PID, proper neuron proportion coefficient, proportion learning speed, integral learning speed and differential learning speed of single neuron PID are gotten by simulation. These coefficients are shown in Table I and II.

Table 1. Parameters of digit PID

\begin{tabular}{|c|c|c|c|}
\hline Type & $K_{P}$ & $K_{I}$ & $K_{D}$ \\
\hline Digit PID & 0.15 & 0.8 & 0.2 \\
\hline
\end{tabular}

Table 2. Parameters of single-neuron self-tuning PID

\begin{tabular}{|c|c|c|c|c|}
\hline Type & $K$ & $\eta_{P}$ & $\eta_{I}$ & $\eta_{D}$ \\
\hline Single neuron self-tuning PID & 0.85 & 0.3 & 0.4 & 0.25 \\
\hline
\end{tabular}

\section{Performance Comparison of Single Neuron Self-Tuning PID Controller and Traditional PID Controller}

A whole control cycle is about $0.2 \mathrm{~s}$ in this paper. When the bath depth is set to $3 \mathrm{~mm}$, as shown in Fig.4 and Fig.5, the simulation curves of single neuron self-tuning PID and traditional PID are gotten, the two controllers can both go stable quickly, and their steady-state errors are both zero, the adjusting time of single neuron PID controller is $2.1 \mathrm{~s}$, and the overshoot is zero; while the adjusting time of traditional PID controller is $3.8 \mathrm{~s}$, the maximal overshoot is about $10 \%$, therefore, the performance of single neuron self-tuning PID controller is better than that of traditional PID controller.

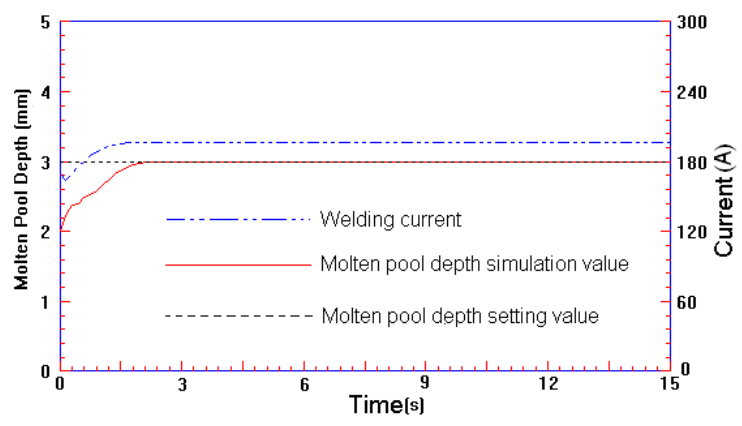

Fig.4 Simulation curves of single neuron self-tuning PID controller (Welding bath depth is set to $3 \mathrm{~mm}$ ) 


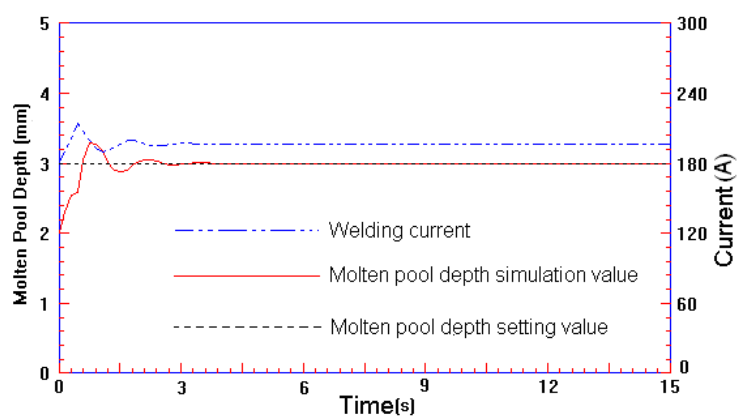

Fig.5 Simulation curves of traditional PID controller (Welding bath depth is set to $3 \mathrm{~mm}$ )

\section{Performance of Single Neuron Self-Tuning PID Controller with Various Learning Algorithms}

When the Welding bath depth is set to $3 \mathrm{~mm}$, as shown in Fig.6, simulation curves of single neuron self-tuning PID controller by using non-supervised Hebb learning rule, supervised Delta learning rule, supervised Hebb learning rule and improved Hebb learning rule are gotten respectively, these controllers all react and go stable quickly, the adjusting times are $3.5 \mathrm{~s}, 2.6 \mathrm{~s}, 2.9 \mathrm{~s}$ and $2.1 \mathrm{~s}$ respectively, their steady-state errors and overshoots are all zeros. When improved Hebb learning is applied, the least time is cost and the performance is best. Therefore, improved Hebb learning rule is selected for single neuron learning in this paper.

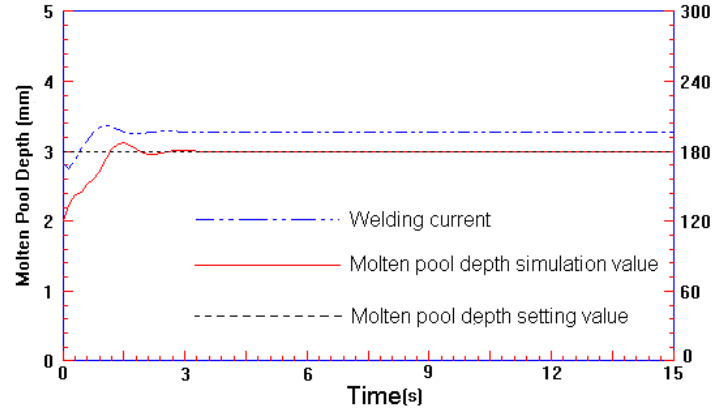

(a)Non-supervised Hebb learning rule

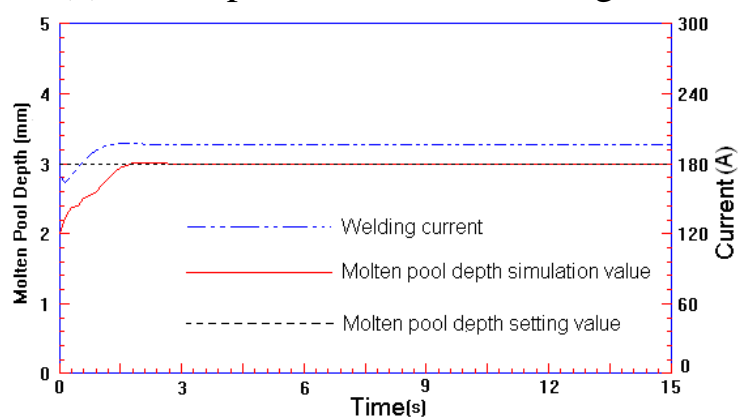

(c)Supervised Hebb learning rule

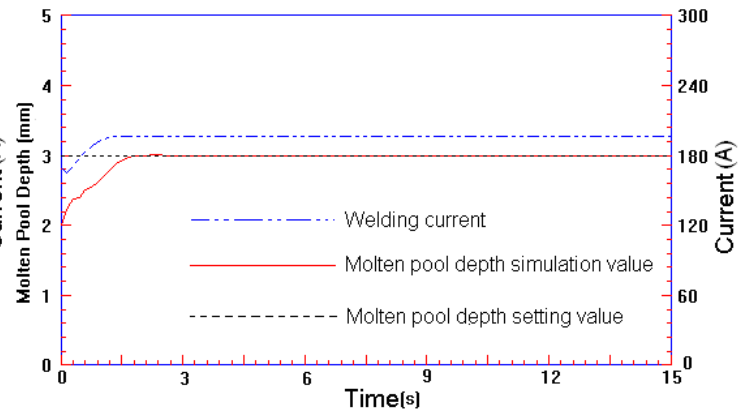

(b) Supervised Delta learning rule

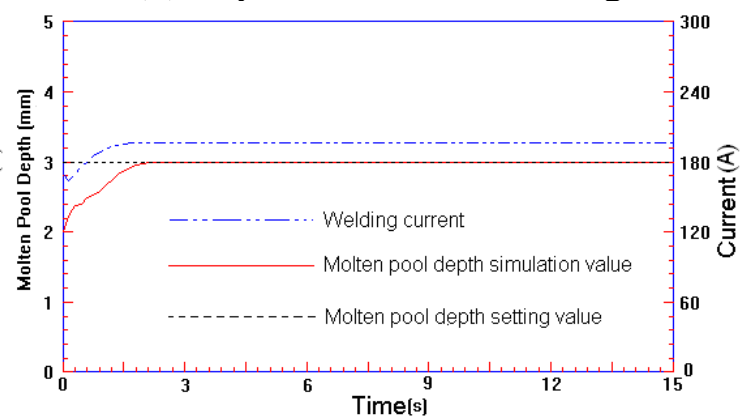

(d) Improved Hebb learning rule

Fig.6 Simulation with various learning rules

\section{Control Experiment}

\section{Experiment Workpiece}

According to the main factors which affect welding bath depth, various cross-section and seam-gap workpiece is used for arc welding experiment. The workpiece's shape is shown in Fig.7, and its thickness is $7 \mathrm{~mm}$. The whole workpiece is divided into four segments which names $\mathrm{AB}, \mathrm{BC}, \mathrm{CD}$ and DE. AB segment is broad, and has 0mm gap. The other segments are narrow. BC, $\mathrm{CD}$ and $\mathrm{DE}$ segments have $0 \mathrm{~mm}, 1 \mathrm{~mm}$ and $2 \mathrm{~mm}$ gap respectively. Every segment is divided into three sub- 
segments labelled as cross-section 1-12. After welding, cut-off the workpiece at each cross-section to get its bath depth. The control target is to keep bath depth stable in welding.

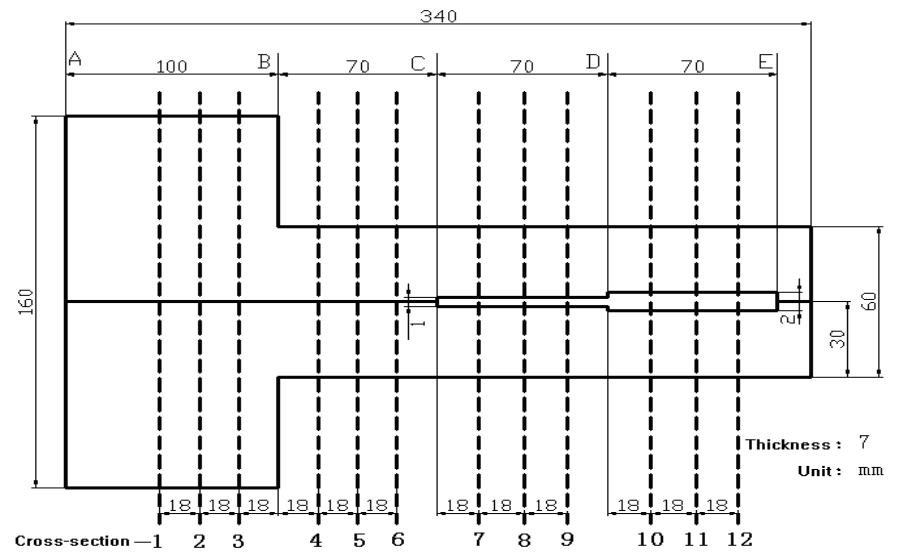

Fig.7 Shape and dimensions of workpiece

\section{Welding Without Control}

Non-control welding is carried out first for comparison, that is to say the welding parameters keep unchanged in the whole process. The curve of cross-sections' molten pool depth after welding is shown in Fig.8.

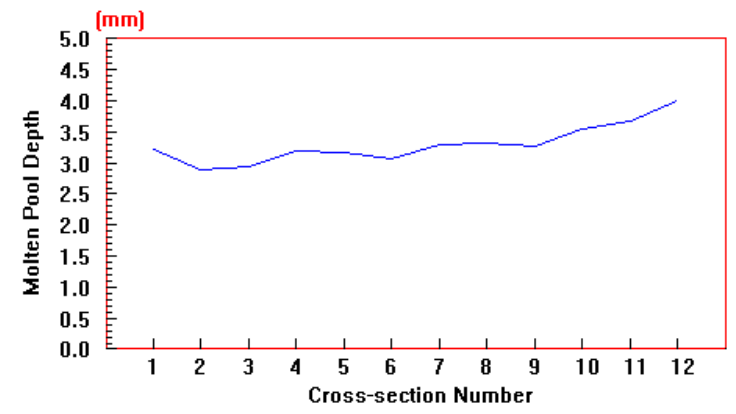

Fig.8 Welding bath depth (Non-control)

From the above figure, it can be seen that the molten pool depth varies greatly, and the difference between the maximal molten pool depth and the minimal molten pool depth is $1.12 \mathrm{~mm}$.

\section{Welding with Single Neuron PID Closed-loop Control}

Welding is carried out by using single neuron self-tuning PID closed-loop control system; the other welding parameters are kept unchanged except welding current. Fig.9 shows the curve of molten pool depth after welding.

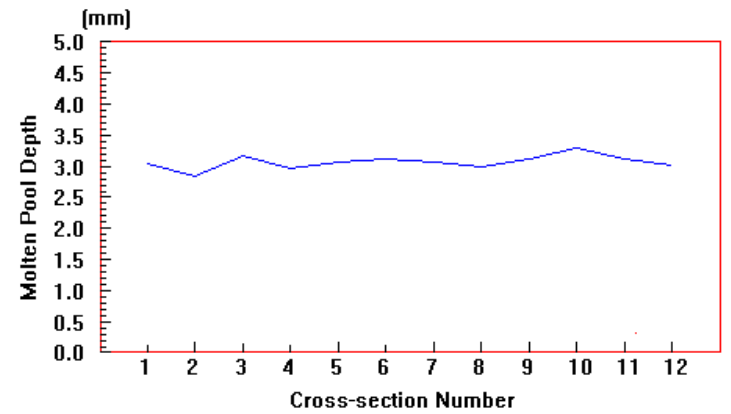

Fig.9 Welding bath depth (Closed-loop Control) 
As shown in Fig.9, in contrast to welding without control, the molten pool depth after closedloop control is much more stable; the difference between the maximal molten pool depth and the minimal bath depth is $0.56 \mathrm{~mm}$, therefore, the precision is increased much.

\section{Conclusion}

1) Simulation results show that best effect can be gotten if improve Hebb learning algorithm is applied to single neuron self-tuning bath depth control system.

2) Welding is a non-linear and time-variant procedure, and single neuron PID controller has simple structure with little calculation and high self-tuning ability. Experiment results show that single neuron self-tuning PID controller is suitable to such complicated systems as welding.

\section{References}

1. L. Wu, S. B. Chen, Intelligent Welding Technology, Beijing: Defence industry press, 2010.

2. S. S. Huang, "Auto-tuning PID control system for molten pool in TIG welding," HANJIE XUEBAO, vol.15, no.2, pp.122 130, June 1994.

3. Z. Zhong, W. Sun, X B. Liu, "Research on single neuron adapting PID controller," Journal of Dalin University of Technology, vol.39, no.5, September 1999.

4. X. Zhu, Y. Q. Hou, J Y. Peng, et al, "Closed-loop control system based on single neuron PID controller," Journal of Northwest University, vol.34, no.4, pp.413 416, August 2004.

5. Z. X. Hou, Q. T. Sheng, Y. H, Wu, "Active suspension system with single neural adaptive PID controller," Journal of system simulation, vol.16, no.9, September 2004.

6. J. K. Liu, et al, Advance PID Control \& MATLAB Simulation, Beijing: electronic industry press, 2014, pp.153-160. 